\title{
Asymmetric Graph Coloring Games
}

H. A. Kierstead

DEPARTMENT OF MATHEMATICS AND STATISTICS

ARIZONA STATE UNIVERSITY

TEMPE, ARIZONA 85287-1804

E-mail: kierstead@asu.edu

Received July 8, 2002; Revised May 17, 2004

Published online in Wiley InterScience(www.interscience.wiley.com).

DOI 10.1002/jgt.20049

Abstract: We introduce the $(a, b)$-coloring game, an asymmetric version of the coloring game played by two players Alice and Bob on a finite graph, which differs from the standard version in that, in each turn, Alice colors a vertices and Bob colors $b$ vertices. We also introduce a related game, the $(a, b)$-marking game. We analyze these games and determine the $(a, b)$ chromatic numbers and $(a, b)$-coloring numbers for the class of forests and all values of $a$ and $b$. ( $) 2005$ Wiley Periodicals, Inc. J Graph Theory 48: 169-185, 2005

Keywords: asymmetric; game coloring number; game chromatic number

\section{INTRODUCTION}

The coloring game is played on a finite graph $G$, using a set $X$ of colors, by two players Alice and Bob with Alice playing first. The players take turns coloring the vertices of $G$ with colors from $X$ so that no two adjacent vertices have the same color. Bob wins if at some time one of the players has no legal move; otherwise Alice wins when the players eventually create a proper coloring of $G$. The game chromatic number of $G$, denoted $\chi_{\mathrm{g}}(G)$, is the least integer $t$ such that Alice has a

(c) 2005 Wiley Periodicals, Inc. 
winning strategy when the game is played on $G$ using $t$ colors. The game chromatic number was first introduced by Bodlaender [1]. Faigle, Kern, Kierstead, and Trotter [4] proved that the game chromatic number of a forest is at most 4. This is best possible as was shown by Bodlaender. Since then many authors have considered game chromatic number and related parameters, including Cai and Zhu [2], Dinski and Zhu [3], Guan and Zhu [5], Kierstead [6], Kierstead and Trotter [7,8], Kierstead and Tuza [9], Nešetřil and Sopena [10], and Zhu [11,12].

While the game model makes it seem that Bob is a malevolent participant in the coloring procedure, this is not the intent. Consider an application in which there are two conflicting sets of requirements. Alice's responsibility is to ensure that the vertices can be properly colored with few colors, while Bob's responsibility is to ensure that the coloring meets some esthetic standard. In ordinary coloring, $100 \%$ of the effort is devoted to using the minimum possible number of colors. However in the coloring game, 50\% of the effort (Alice's part) is devoted to minimality while the remaining $50 \%$ of the effort (Bob's part) can be devoted to esthetics. Here we consider the effect of varying these percentages.

More formally, we consider a variant of the coloring game in which Alice and Bob are allowed to make several moves in a row. The $(a, b)$-coloring game is played like the coloring game with the exception that on each turn Alice colors $a$ vertices and Bob colors $b$ vertices. (If there are no uncolored vertices left, the players are not required to complete their turns.) Thus the $(1,1)$-coloring game is just the coloring game. The $(a, b)$-game chromatic number of $G$, denoted $\chi_{\mathrm{g}}(G ; a, b)$, is the least integer $t$ such that Alice has a winning strategy when the $(a, b)$-coloring game is played on $G$ using $t$ colors.

Marking games are simplified versions of coloring games that have proved useful in bounding the game chromatic number. A marking game is played by two players Alice and Bob with Alice playing first. At the start of the game, all vertices are unmarked. A play by either player consists of marking an unmarked vertex. The game ends when all the vertices have been marked. For any $t \in\{1, \ldots,|V|\}$, let $M^{t}$ denote the set of marked vertices after $t$ plays and $U^{t}=V-M^{t}$ denotes the set of unmarked vertices after $t$ plays. So $\left|M^{t}\right|=t$. For an unmarked vertex $u$, let $S^{t}(u)=N_{G}(u) \cap M^{t}$. The score of the marking game is

$$
\max \left\{\left|S^{t}(u)\right|: 1 \leq t \leq|V| \wedge u \in U^{t}\right\}
$$

Zhu [11] defined the game coloring number, $\operatorname{col}_{\mathrm{g}}(G)$, of $G$ to be the least $s$ such that Alice has a strategy that results in a score strictly less than $s$ in the marking game. In later work, Nešetrril and Sopena [10] defined the Go number of $G$ to be one less than the game coloring number of $G$.

A play of a marking game determines a linear ordering on the vertices of $G$ in which $x<y$ if $x$ is marked before $y$. The importance of the game coloring number of $G$ is that if Alice uses the strategy for the marking game on $G$ that guarantees a score of $\operatorname{col}_{\mathrm{g}}(G)$ to choose vertices to color, then she can win the coloring 
game using a set of $\operatorname{col}_{\mathrm{g}}(G)$ colors just by coloring with First-Fit. It follows easily that

$$
\chi_{\mathrm{g}}(G) \leq \operatorname{col}_{\mathrm{g}}(G)
$$

Faigle et al. [4] actually bounded the game chromatic number of forests by showing that the game coloring number of a forest is at most 4.

We will also consider the $(a, b)$-marking game. In this variation, Alice plays by marking $a$ vertices and Bob plays by marking $b$ vertices. The $(a, b)$-game coloring number, $\operatorname{col}_{\mathrm{g}}(G ; a, b)$, is the least $s$ such that Alice has a strategy that results in a score strictly less than $s$ in the $(a, b)$-marking game. Here we must be a little careful. The score is computed each time a player marks a vertex, not just at the end of the turns. It then follows that

$$
\chi_{\mathrm{g}}(G ; a, b) \leq \operatorname{col}_{\mathrm{g}}(G ; a, b)
$$

For a class of graphs $\mathcal{C}$ let

$$
\chi_{\mathrm{g}}(\mathcal{C} ; a, b)=\max _{G \in \mathcal{C}} \chi_{\mathrm{g}}(G ; a, b) \text { and } \operatorname{col}_{\mathrm{g}}(\mathcal{C} ; a, b)=\max _{G \in \mathcal{C}} \operatorname{col}_{\mathrm{g}}(G ; a, b) .
$$

In this paper, we will determine the $(a, b)$-game chromatic and $(a, b)$-game coloring numbers for the class $\mathcal{F}$ of forests and all values of $a$ and $b$. Our motivation for studying this class is twofold. First, it is a simple enough class that we can obtain exact results. Second, there is a long history of game chromatic results for forests being extended in non-trivial ways to more complicated graphs. We shall prove the following theorem.

Theorem 1. Let $a$ and $b$ be positive integers.

(a) If $a<b$ then $\chi_{\mathrm{g}}(\mathcal{F} ; a, b)=\operatorname{col}_{\mathrm{g}}(\mathcal{F} ; a, b)=\infty$.

(b) If $b \leq a$ then $b+2 \leq \chi_{\mathrm{g}}(\mathcal{F} ; a, b) \leq \operatorname{col}_{\mathrm{g}}(\mathcal{F} ; a, b) \leq b+3$.

(c) If $b \leq a<\max \{2 b, 3\}$ then $b+3 \leq \chi_{\mathrm{g}}(\mathcal{F} ; a, b)$.

(d) If $4 \leq 2 b \leq a<3 b$ then $\chi_{\mathrm{g}}(\mathcal{F} ; a, b) \leq b+2<b+3 \leq \operatorname{col}_{\mathrm{g}}(\mathcal{F} ; a, b)$.

(e) If $3 b \leq a$ then $\operatorname{col}_{\mathrm{g}}(\mathcal{F} ; a, b) \leq b+2$.

There are several little surprises hidden in the statement of this theorem. There are not many interesting examples of classes of graphs for which the known upper bounds on the game chromatic number and the game coloring number differ. Indeed, most interesting upper bounds on the game chromatic number are proved by showing the same bound for the game coloring number. In the case of forests, we actually have $\chi_{\mathrm{g}}(\mathcal{F})=\operatorname{col}_{\mathrm{g}}(\mathcal{F})$. A careful reading of the theorem indicates that $\chi_{\mathrm{g}}(\mathcal{F} ; 2,1)=\operatorname{col}_{\mathrm{g}}(\mathcal{F} ; 2,1)$. At first we were misled by this fact. However, this equality between $\chi_{\mathrm{g}}$ and $\operatorname{col}_{\mathrm{g}}$ does not hold in general. Specifically, Theorem 1 yields the following exact values. 
Corollary 2. Let $a$ and $b$ be positive integers.

(a) If $b \leq a<2 b$, or if $(a, b)=(2,1)$ then $\chi_{\mathrm{g}}(\mathcal{F} ; a, b)=\operatorname{col}_{\mathrm{g}}(\mathcal{F} ; a, b)=$ $b+3$.

(b) If $2 b \leq a<3 b$ and $b>1$ then $\chi_{\mathrm{g}}(\mathcal{F} ; a, b)=b+2$ and $\operatorname{col}_{\mathrm{g}}(\mathcal{F} ; a, b)=$ $b+3$.

(c) If $3 b \leq a$ then $\chi_{\mathrm{g}}(\mathcal{F} ; a, b)=\operatorname{col}_{\mathrm{g}}(\mathcal{F} ; a, b)=b+2$.

Of course, these results are most interesting for small values of $a$ and $b$. The same results hold if the class of forests is replaced by the class of trees.

This paper is organized as follows. In Section 2, we prove all upper bounds of Theorem 1. The upper bounds of (b), (d), and (e) follow from Lemmas 2, 4, and 3, respectively. In Section 3, we prove all lower bounds. The lower bounds for (a), (b), (c), and (d) follow from Lemmas 9, 6, 7 and 8, and 10, respectively. We end this section by reviewing our notation.

For any positive integer $n$, let $[n]$ denote the set $\{1,2, \ldots, n\}$. Let $\lambda$ denote the empty sequence and $S^{i}$ denote the set of $i$-sequences whose entries are chosen from $S$. Two functions $f: A \rightarrow B$ and $g: C \rightarrow D$ are compatible if $f(x)=g(x)$ for all $x \in A \cap C$. In this case, $f \cup g$ denotes the function on $A \cup C$ that extends both $f$ and $g$. For $A_{0} \subseteq A$, we write $f \mid A_{0}$ for the restriction of $f$ to $A_{0}$ and $f\left[A_{0}\right]$ for the range of $f \mid A_{0}$. Let $G=(V, E)$ be a graph. If $W \subseteq V$ we will sometimes write $W$ to denote the graph $G[W]$ induced in $G$ by $W$. If $v$ is a vertex of $G$, then $N_{G}(v)=\{x \in V: v x \in E\}$ denotes the neighborhood of $v$. The degree of $v$ is $d_{G}(v)=\left|N_{G}(v)\right|$. For a subset $S$ of $V$, let $N_{G}(S)=\bigcup\left\{N_{G}(v)-S: v \in S\right\}$. If $G$ is clear from the context, we may drop the subscripts. The distance, $\operatorname{dist}(x, y)$, between two vertices $x$ and $y$ is the number of edges in the shortest path between $x$ and $y$. If, for example, the value of $a$ is to be replaced by $a+1$, we will write $a \leftarrow a+1$.

\section{UPPER BOUNDS}

In this section, we prove upper bounds on $\chi_{\mathrm{g}}(\mathcal{F} ; a, b)$ and $\operatorname{col}_{\mathrm{g}}(\mathcal{F} ; a, b)$. Not surprisingly, our proofs are based on a proof that $\operatorname{col}_{\mathrm{g}}(T) \leq 4$, for any forest $T$. There are two proofs of this result. The first [4] has given rise to the very general activation strategy that Alice can use successfully on a wide variety of graphs, including planar graphs. A second, easier proof [9] has not been as useful. But it is this second proof that we exploit here. For completeness and to motivate the harder proofs that follow, we include this basic proof in the proof of Lemma 3.

For the rest of this section, let $T=(V, E)$ be any fixed forest. We will show that Alice has winning strategies for various versions of the game played on $T$. At any time in the game, let $M$ be the set of vertices that have been marked and $U=V-M$ be the set of unmarked vertices. A component of $U$ is called an 
unmarked component. Let $S$ be an unmarked component. Then $N(S) \subseteq M$, by our definition of $N(S)$. The weight $w(S)$ of $S$ is the number $w(S)=|N(S)|$ of marked vertices adjacent to unmarked vertices in $S$. Note that, since $S$ is a tree, if one of the players marks a vertex $v \in S$ such that $N(v) \cap M \neq \emptyset$, then each component $S^{\prime}$ of $S-\{v\}$ has weight $w\left(S^{\prime}\right) \leq w(S)$.

Lemma 3. If $b \leq a$, then $\operatorname{col}_{\mathrm{g}}(\mathcal{F} ; a, b) \leq b+3$.

Proof. We will show that Alice has a strategy that results in a score of at most $b+2$ in the $(a, b)$-marking game on $T$. We first show that $\operatorname{col}_{\mathrm{g}}(T ; 1,1) \leq 4$. Alice will play so as to maintain the following invariant: At the end of each of Alice's plays, the weight of any unmarked component is at most 2 . If she achieves this goal, then no unmarked vertex is ever adjacent to more than three marked vertices. Indeed, after Alice's play, no unmarked vertex is adjacent to more than two marked vertices, and after Bob's next play no unmarked vertex is adjacent to more than three marked vertices.

On her first play Alice marks any vertex and the invariant clearly holds. Now suppose that the invariant held after Alice's last play and Bob has just marked a vertex $v$ in a component $S$ of $V-M$. Suppose $S^{\prime}$ is now an unmarked component of $V-(M \cup\{v\})$ with weight 3. Then $S^{\prime} \subset S$ and $N(S) \cup\{v\}=N\left(S^{\prime}\right)$. Since $T$ is a forest, two marked vertices cannot each be adjacent to vertices in the same two unmarked components. Thus there is at most one component $S^{\prime}$ of $V-(M \cup\{v\})$ with weight 3. It is easy to check that there exists a vertex $u \in S^{\prime}$ such that after $u$ is marked, each component of $S^{\prime}-\{u\}$ has weight at most 2. So if there is an unmarked component with weight 3 , Alice chooses it; otherwise she chooses any component of $V-(M \cup\{v\})$. Once she has chosen an unmarked component $S^{*}$, she marks a vertex $u \in S^{*}$, such that each component of $S^{*}-\{u\}$ has weight at most 2.

Notice that Alice could still use the above strategy if Bob is allowed to pass on some turns. It follows that $\operatorname{col}_{\mathrm{g}}(T ; a, 1) \leq 4$. Finally, consider the $(a, b)$-marking game. We will show that Alice can still maintain the invariant. Then after any of her turns, no unmarked vertex is adjacent to more than two marked vertices. It follows that after any of Bob's turns, no unmarked vertex is adjacent to more than

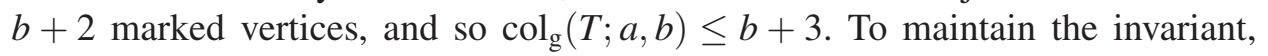
Alice simulates the $(1,1)$-marking game. She considers the set $M$ of marked vertices after her last turn together with the last $b$ vertices $v_{1}, \ldots, v_{b}$ that Bob has marked. First she sets $i:=1$. Then she asks herself where she would have played in the (1,1)-marking game if Bob had just marked $v_{i}$. Then $M \leftarrow M \cup\left\{v_{i}\right\}$. If her response would be $u_{i}$ then she checks to see whether $u_{i} \in\left\{v_{i+1}, \ldots, v_{b}\right\}$. If so, by reordering if necessary, assume $u_{i}=v_{i+1}$. In this case, Bob has answered his own threat, and she resets $M \leftarrow M \cup\left\{v_{i+1}\right\}$ and $i \leftarrow i+2$. Otherwise she marks $u_{i}$ and resets $M \leftarrow M \cup\left\{u_{i}\right\}$ and $i \leftarrow i+1$. Continuing in this fashion until $i>b$, she marks vertices when necessary to respond to threats by Bob that he does not answer himself. Eventually, when $i>b$, she will have marked at most $b \leq a$ vertices. If she has not yet marked $a$ vertices she continues as though Bob was 
playing by passing in the (1,1)-marking game. In this way, she maintains the invariant.

Next we show that if $a$ is large enough then the above bound can be slightly improved.

Lemma 4. If $3 b \leq a$, then $\operatorname{col}_{\mathrm{g}}(\mathcal{F} ; a, b) \leq b+2$.

Proof. We will show that Alice has a strategy that results in a score of at most $b+1$ in the $(a, b)$-marking game played on $T$. Suppose that $S$ is an unmarked component with weight 2 . Then there exist two marked vertices $x$ and $y$ and a unique $x-y$ path $P_{S}$ in $T$ with at least three vertices such that all internal vertices of $P_{S}$ are in $S$. Alice's strategy will be to play so that immediately after her play, the following properties hold for each unmarked component $S \subset U$ :

(1) $w(S) \leq 2$.

(2) If $w(S)=2$, then either $|S|=1$ or $\operatorname{dist}(x, y)$ is odd.

Moreover, Alice will always mark a vertex $v$ in an unmarked component $S$ such that:

(3) either $w(S)=0$ or $v$ is adjacent to a marked vertex or $v$ is on a path between two marked vertices.

Notice that (1)-(3) imply that an unmarked vertex is never adjacent to $b+2$ marked vertices. Indeed, after Alice's play this follows from (1). Moreover, by (2) the only way that an unmarked vertex can be adjacent to two marked vertices after Alice's play is that it has no unmarked neighbors. Thus after Bob's next play no unmarked vertex can have $b+2$ marked neighbors. By (3), no unmarked component with positive weight can ever gain weight while Alice is playing. In particular, no unmarked vertex can ever have $b+2$ marked neighbors.

Alice's strategy is somewhat complicated by the requirement that she use all $a$ of her moves if there are unmarked vertices remaining. Suppose that at a certain time both (1) and (2) hold. We claim that she can safely make two more moves, satisfying (3) and maintaining (1) and (2). First, if each unmarked component has size 1, Alice marks any two unmarked vertices (or one, if only one remains). Otherwise, suppose that there exists an unmarked component $S$ with $|S| \geq 2$. If $w(S) \leq 1$, then Alice marks a vertex $v \in S$ such that if $w(S)=1$, then $v$ has a neighbor in $M$. Each of the (at least 1) components of $S-v$ has weight 1 , so Alice can now safely mark a vertex $w \in S$ that is adjacent to $v$, leaving all unmarked components contained in $S$ with weight 1 . Otherwise $w(S)=2$. Let $\{x, y\}=N(S)$ and $P_{S}=x x_{1} x_{2} \ldots x_{n-1} y$. By (2) and the assumption that $|S| \geq 2$, it follows that $n>1$ is odd. Alice can safely mark $x_{1}$ and then $x_{n-1}$. Then all components $S^{\prime} \subseteq S-\left\{x_{1}, x_{n-1}\right\}$ have weight at most 2 . If $w\left(S^{\prime}\right)=2$, then $N\left(S^{\prime}\right)=\left\{x_{1}, x_{n-1}\right\}$ and the unique $x_{1}-x_{n-1}$ path has odd length. 
We first consider the case that $b=1$ and argue by induction on $a$. By the above remarks, if Alice can maintain (1)-(3) with $a=k$, then she can maintain (1)-(3) with $a=k+2$. The only difficulty is in the base steps $a=3$ and 4 . First suppose that $a=3$. It suffices to show that Alice can play satisfying (3) and maintaining (1) and (2) after either three moves, or, using the previous argument, one move. We consider the position at the start of Alice's turn. There are three possibilities.

Position 1. (1) and (2) both hold. If there exists an unmarked component $S$ with $w(S) \leq 1$ or $|S|=1$, then Alice can safely mark a vertex $v \in S$ such that if $w(S)=1$, then $v$ has a neighbor in $M$. Otherwise there exists an unmarked component $S$ such that $w(S)=2$ and $|S| \geq 2$. Let $N(S)=\{x, y\}$ and $P_{S}=$ $x x_{1} x_{2} \ldots x_{n-1} y$. By (2) $n$ is odd, and so $n \geq 3$. If $d_{U}\left(x_{1}\right) \geq 2$, then Alice marks $x_{1}$, then $x_{2}$, and finally $z \in N\left(x_{1}\right) \cap\left(S-\left\{x_{2}\right\}\right)$. Then $S-\left\{x_{1}, x_{2}, z\right\}$ has one component $S^{\prime}$ that contains $\left\{x_{3}, \ldots, x_{n-1}\right\}$, and possibly some other unmarked components of weight 1 . Only $S^{\prime}$ has weight 2 . Since $P_{S^{\prime}}=x_{2} \ldots x_{n-1} y$ has odd length, $S^{\prime}$ satisfies 2. If $d_{U}\left(x_{1}\right)=1$, then Alice marks $x_{2}$. This breaks $S-\left\{x_{2}\right\}$ up into an unmarked component $S_{0}=\left\{x_{1}\right\}$, an unmarked component $S_{1}$ that contains $x_{3} \ldots x_{n-1}$, and possibly some other unmarked components. Only $S_{0}$ and $S_{1}$ have weight 2 . Both satisfy (2) since $\left|S_{0}\right|=1$ and $P_{S_{1}}=x_{2} \ldots x_{n-1} y$ has odd length.

Position 2. (1) fails, that is (since $b=1$ ), there exists an unmarked component $S \subset U$ with $w(S)=3$. Let $N(S)=\{x, y, z\}$. After Alice's last move (1) held, so Bob has just marked one of these vertices, say $z$. After Alice's last move $z$ was in some unmarked component $S_{0}$. So $S \subset S_{0}-\{z\}$ and $N\left(S_{0}\right)=\{x, y\}$. Any other unmarked component contained in $S_{0}-z$ has weight 1. Any unmarked component not contained in $S_{0}-z$ still satisfies (1) and (2) (since $b=1$ ). So it suffices for Alice to play (satisfying (3)) by marking vertices in $S$ so that the resulting unmarked components satisfy (1) and (2). By (2) applied to $S_{0}$, $P_{S_{0}}=x v_{1} \ldots v_{n-1} y$ has odd length. Clearly $z$ is not on $P_{S_{0}}$. Let $v_{i}$ be the closest vertex on $P_{S_{0}}$ to $z$ and $Q=z z_{1} \ldots z_{t-1} v_{i}$ be the unique $z-P_{S_{0}}$ path. Without loss of generality, $\operatorname{dist}\left(x, v_{i}\right)=i$ is odd and $\operatorname{dist}\left(y, v_{i}\right)=n-i$ is even. Alice first marks $v_{i}$. This leaves at most three unmarked components of $S-\left\{v_{i}\right\}$ with weight 2: the component $S_{1}$ with $x \in N\left(S_{1}\right)$, the component $S_{2}$ with $y \in N\left(S_{2}\right)$, and the component $S_{3}$ with $z \in S_{3}$. If $i=1$, then $S_{1}=\emptyset$ and if $t=1$, then $S_{3}=\emptyset$. However, since $\operatorname{dist}\left(y, v_{i}\right)$ is even, $S_{2}$ contains at least one vertex $v_{i+1}$. All other components of $S-\left\{v_{i}, v_{i+1}, z_{t-1}\right\}$ have weight 1 .

Position 2a. The length of $Q$ is even. Then $S_{3} \neq \emptyset$. Alice plays by marking $v_{i+1} \in S_{2}$ and $z_{t-1} \in S_{3}$. Since each of the paths $P_{S_{1}}=x v_{1} \ldots v_{i-1}, P_{S_{2}}=v_{i+2} \ldots$ $v_{n-1} y$, and $P_{S_{3}}=z z_{1} \ldots z_{t-1}$ is odd, (2) is satisfied.

Position 2b. The length of $Q$ is odd. Since $P_{S_{1}}=x v_{1} \ldots v_{i-1}$ and $P_{S_{3}}=$ $z z_{1} \ldots z_{t-1} v_{i}$ are odd, $S_{1}$ and $S_{3}$ satisfy (2). However, Alice still must deal with $S_{2}$. If $d_{S}\left(v_{i+1}\right)>2$, then she marks $v_{i+1}$ and $w \in N_{S}\left(v_{i+1}\right)-\left\{v_{i}, v_{i+2}\right\}$. This results in at most one new component $S^{\prime}$ with weight 2 . Then $P_{S^{\prime}}=v_{i+2} \ldots v_{n-1} y$ has odd 
length, so (2) is satisfied. Otherwise, $d_{S}\left(v_{i+1}\right)=2$. If $i=n-2$, then $\left|S_{2}\right|=1$ and we are done, since $S_{2}$ satisfies (2) already. If not, Alice marks $v_{i+2}$ and $v_{i+3}$. This leaves at most two new components of $S_{2}-\left\{v_{i+2}, v_{i+3}\right\}$ with weight 2: the component $S^{\prime}=\left\{v_{i+1}\right\}$ and the component $S^{\prime \prime}$ that contains $v_{n-1}$. (If $n=i+4$, then $S^{\prime \prime}=\emptyset$.) Since $\left|S^{\prime}\right|=1, S^{\prime}$ satisfies (2), and since $P_{S^{\prime \prime}}=v_{i+3}, \ldots, v_{n-1} y$ is odd, $S^{\prime \prime}$ satisfies (2).

Position 3. (1) holds for all unmarked components $S$, but there exists an unmarked component $S$ such that (2) fails. There can only be one such component, since $b=1$. Then $P_{S}=x v_{1}, \ldots, v_{n-1} y$ has even length. Alice satisfies (1) and (2) by marking $v_{1}$.

This completes the proof when $a=3$. Next we check that with minor modifications, Alice's strategy also works when $a=4$. Position 1 is easy by our introductory remarks, since 4 is even. Also Position 3 is easy: Alice marks $v_{1}$ to obtain Position 1 with three remaining moves. Thus she is done by the case $a=3$. So we are left with Position 2. Position $2 \mathrm{~b}$ is now easier: after Alice marks $v_{i}$ and $v_{i+1}$, both (1) and (2) hold. In Position 2a, Alice first marks $z_{t-1}$. This leaves Position $2 \mathrm{~b}$ and she is again done by the case $a=3$. This completes the case $b=1$.

Finally we consider the case $b>1$. To maintain (1) and (2), Alice simulates the (3,1)-marking game. She considers the set $M$ of marked vertices after her last turn together with the last $b$ vertices $v_{1}, \ldots, v_{b}$ that Bob has marked. First she asks herself where she would have played in the $(3,1)$-marking game if Bob had just marked $v_{1}$. Then $M \leftarrow M \cup\left\{v_{1}\right\}$. If her response would be $u, v, w$, then she marks the vertices in the set $X=\{u, v, w\}-\left\{v_{2}, \ldots, v_{b}\right\}$. Let $a^{\prime}=a-|X|$ and $b^{\prime}=b-4+|X|$. Note that $3 b^{\prime} \leq a^{\prime}$. Now the situation is the same as if Alice was playing the $\left(a^{\prime}, b^{\prime}\right)$-marking game and Bob had just marked the vertices in the set $\left\{v_{2}, \ldots, v_{b}\right\}-\{u, v, w\}$. Thus, arguing inductively, Alice can play so as to maintain (1)-(3).

Finally, we show that Alice has an improved strategy for the $(a, b)$-coloring game on forests. We will need to modify the definition of weight now that we are dealing with colored vertices rather than marked vertices. Given a forest $T=$ $(V, E)$, suppose that $c: C \rightarrow X$ is a proper coloring with $C \subseteq T$ and $S$ is a component of $T-C$. (So $N(S) \subseteq C$.) For an uncolored vertex $u \in S$, define the weight $w(u)$ of $u$ by $w(u)=|\{c(v): v \in C \cap N(u)\}|$ and the weight $w(S)$ of $S$ by $w(S)=\sum_{u \in S} w(u)$. Thus $w(u)$ is the number of colors used on vertices in $C \cap N(u)$. Also

$$
|\{c(v): v \in N(S)\}| \leq w(S) \leq|N(S)|
$$

Let $i \cong j$ denote that $i \equiv j(\bmod 3)$. Call a pair $\{x, y\} \subseteq C$ well colored by $c$ if $c(x)=c(y)$ if and only if $\operatorname{dist}(x, y) \cong 2$. A component $S$ of $T-C$ is well bounded by $c$ if $w(S) \leq 2$ and all pairs $\{x, y\} \subseteq N(S)$ are well colored. The coloring $c$ is good if $c$ is proper and all components of $T-C$ are well bounded by $c$. 
Lemma 5. If $4 \leq 2 b \leq a$, then $\chi_{\mathrm{g}}(\mathcal{F} ; a, b) \leq b+2$.

Proof. We must show that Alice has a winning strategy in the $(a, b)$-coloring game on $T=(V, E)$ using the set $X=[b+2]$ of colors. It suffices to show that Alice can always play so that immediately after her turn the coloring $c: C \rightarrow X$ that the players have produced is good. Then, in particular, all the colored neighbors of any uncolored vertex have been colored with the same color. It follows that Bob cannot win on his next play.

First we show that if $c: C \rightarrow X$ is a good coloring, then there is a good extension of $c$ to $c^{\prime}: C \cup\{u\} \rightarrow X$ for some uncolored vertex $u$. Let $S$ be a component of $T-C$ and $u \in S$ be adjacent to a colored vertex $x \in C$. Color $u$ with a color different from $c(x)$. Moreover, if $w(S)=2$ and $y \in N(S-\{u\}) \cap C$, then color $u$ so that $c^{\prime}(u)=c(y)$ if and only if $\operatorname{dist}(u, y) \cong 2$. This is possible since $\{x, y\}$ is well colored and so $\operatorname{dist}(u, y) \cong 2$ if and only if $\operatorname{dist}(x, y) \cong 0$. Thus if Alice obtains a good coloring before coloring $a$ vertices, she can still complete her turn while maintaining a good coloring.

As in the marking game, when Bob colors vertices in a well bounded, uncolored component $S$, Alice will try to process vertices so that $S$ is broken up into several new, well bounded, uncolored components. In the marking game, Bob's ability to color multiple vertices proved to be only a minor annoyance, because if his moves got in the way of Alice's intended moves, she could pretend that they were her own. This is no longer the case in the coloring game. Alice may want to color a vertex with a particular color, but find that Bob has either already colored that vertex with a different color or has colored a neighbor of it with the same color.

On his last turn, Bob may have colored vertices in several uncolored components. Alice will repair any damage that he has done in these components one at a time. Consider any uncolored component $S$, its neighborhood $N=N(S)$, and the set $B \subseteq S$ of vertices in $S$ that Bob colored on his last turn. Let $c: N \rightarrow X$ be the coloring of $N$ at the start of Bob's last turn and $c_{b}: N \cup B \rightarrow X$ be the coloring of $N \cup B$ at the end of Bob's last turn. Alice will repair any damage that Bob has done by coloring a set $A \subseteq S-B$ of at most $2|B|$ of the (at least) $2 b$ vertices that she is allowed to color on her next turn to create a good coloring $c_{a}: N \cup B \cup A \rightarrow X$ that extends $c_{b}$. More formally, it suffices to prove the following lemma.

Lemma 6. Let $S$ be a proper subtree of a tree $T, N=N(S)$, and $c: N \rightarrow X$ be a good coloring. For every subset $B \subseteq S$ with $|B| \leq b$ and proper coloring $c_{b}: N \cup B \rightarrow X$ extending $c$, there exists a subset $A \subseteq S-B$ with $|A| \leq 2|B|$ and a good coloring $c_{a}: N \cup B \cup A \rightarrow X$ extending $c_{b}$.

Proof. We argue by induction on $|B|$. The base step $B=\emptyset$ is trivial, so consider the induction step $B \neq \emptyset$. By the induction hypothesis, it suffices to show that there exist subsets $B^{\prime} \subseteq B$ and $A^{\prime} \subseteq S-B$ and a coloring $c^{\prime}: N \cup$ $A^{\prime} \cup B^{\prime} \rightarrow X$ extending $c_{b} \mid B^{\prime}$ such that 
(1) $B^{\prime} \neq \emptyset$ and $\left|A^{\prime}\right| \leq 2\left|B^{\prime}\right|$.

(2) $c^{\prime}$ is a good coloring.

(3) $c_{b} \cup c^{\prime}$ is a proper coloring.

The condition that $A^{\prime} \subseteq S-B$ ensures that $c^{\prime}$ is compatible with $c_{b}$. By (1) we make progress without using up too many vertices. We will consider several possible cases. Clearly $w(S) \neq \emptyset$.

Case 0. $w(S)=1$ (with respect to $c$ ). Let $P=\left(x=x_{0} x_{1} \ldots x_{n}=y\right.$ ) be a shortest path from a vertex in $N$ to a vertex in $B$ and set $B^{\prime}=\{y\}$. Let $s \cong n$, where $s \in\{0,1,2\}$. Let $\Gamma=X-c_{b}[B \cup\{x\}]$ be the set of colors in $X$ that do not appear on any vertex in $B \cup\{x\}$. Then $\Gamma \neq \emptyset$, since $w(S) \leq 1$. First suppose that $s \neq 0$. Let $A^{\prime}=\left\{x_{1}\right\}$ and let $c^{\prime}\left(x_{1}\right)=\beta$, where $\beta \in \Gamma$. Clearly (3) holds. The only component (if any) of $S-\left(A^{\prime} \cup B^{\prime}\right)$ that has weight at least two is the component that contains $x_{2}$. Since $\operatorname{dist}\left(x_{1}, y\right) \neq 2$ and $c^{\prime}\left(x_{1}\right) \neq c^{\prime}(y)$, this component is well bounded and $c^{\prime}$ is good. Otherwise, $s=0$. Let $A^{\prime}=\left\{x_{1}, x_{2}\right\}$. Since $c$ is a good coloring $c(x) \neq c(y)$. Set $c^{\prime}\left(x_{2}\right)=\alpha$, where $\alpha \in \Gamma$. Next set $c^{\prime}\left(x_{1}\right)=\beta$, where $\beta \in \Gamma-\{\alpha\}$ if $|\Gamma|>1$ and $\beta=c(y)$ otherwise. Note that if $|\Gamma|=1$, then $y$ is the only vertex of $B \cup\{x\}$ that is colored with $\beta$. It follows that in either case, $c_{b} \cup c^{\prime}$ is a proper coloring. Also the only component of $S-\left(A^{\prime} \cup B^{\prime}\right)$ with weight greater than 1 is the component that contains $x_{3}$. Since $\operatorname{dist}\left(x_{2}, y\right) \cong 1$ and $c^{\prime}\left(x_{2}\right) \neq c^{\prime}(y)$, this component is well bounded and $c^{\prime}$ is good.

So suppose that Case 0 fails. Then $w(S)=2$. Let

$$
P=\left(x=x_{0} x_{1} \ldots x_{n}=y\right)
$$

be a path between two vertices of $N$ that is as long as possible. Since $w(S)=2$, there is only one choice for the internal vertices of $P$. Since $c$ is good, there is only one choice for the color of the endpoints of $P$. So neither the length of $P$ nor the colors of its endpoints depend on the choice of endpoints for $P$. Let $\Gamma=X-c_{b}[B \cup\{x, y\}]$. Since $c$ is good, $\Gamma=X-c_{b}[B \cup N]$. Note that $\Gamma \neq \emptyset$ if two vertices in $B \cup\{x, y\}$ have the same color. In particular, if $x$ and $y$ have a common neighbor, then $\Gamma \neq \emptyset$. For each $v \in B$, let $Q_{v}$ be the path in $T$ from $v$ to $P$.

Case 1. Case 0 fails, but there exists $i \in\{0,1, \ldots, n-1\}$ such that $x_{i}, x_{i+1} \in B \cup\{x, y\}$. Let $S^{1}$ and $S^{2}$ be the two components obtained from $S$ by removing the edge $x_{i} x_{i+1}$. Each is empty or has weight 1 with respect to $c$. Since $N\left(S^{1}\right) \cap N\left(S^{2}\right)=\emptyset$, we are done by Case 0 applied to $S^{1}$ and $S^{2}$.

Note that if Case 1 fails then the neighbors in $P$ of any vertex in $B \cap P$ are eligible for $A^{\prime}$; this is important in what follows.

Case 2. Cases 0 and 1 fail, but there exists $i \in[n-1]$ such that $x_{i} \in B$. Let $s, t \in\{0,1,2\}$ satisfy $s \cong \operatorname{dist}\left(x, x_{i}\right)$ and $t \cong \operatorname{dist}\left(y, x_{i}\right)$. If possible, choose $i$ so that $\left\{x, x_{i}\right\}$ is well colored if and only if $\left\{x_{i}, y\right\}$ is well colored. If this is not 
possible, try to choose $x_{i}$ so that if $\left\{x, x_{i}\right\}$ is not well colored then $s \neq 0$ and if $\left\{x_{i}, y\right\}$ is not well colored then $t \neq 0$.

First suppose that both $\left\{x, x_{i}\right\}$ and $\left\{x_{i}, y\right\}$ are well colored. Set $A^{\prime}=\emptyset$, $B^{\prime}=\left\{x_{i}\right\}$, and $c^{\prime}=c_{b} \mid N \cup B^{\prime}$. Then $c^{\prime}$ is good and $c_{b} \cup c^{\prime}=c_{b}$ is proper.

Next suppose that neither $\left\{x, x_{i}\right\}$ nor $\left\{x_{i}, y\right\}$ is well colored. Since $\{x, y\}$ is well colored, $c(x)=c(y)$ iff $s+t \cong 2$. Without loss of generality, suppose that $s \geq t$. Consider the possibility that $t=0$. If $s=2$ then $c(x)=c(y)$, but $c(x) \neq c\left(x_{i}\right)=$ $c(y)$, which is a contradiction. Similarly, if $s \neq 2$ then $c(x) \neq c(y)$, but $c(x)=$ $c\left(x_{i}\right)=c(y)$, which is another contradiction. We conclude that $t>0$. Thus either (i) $s=1=t$ or (ii) both $s=2$ and $t \neq 0$. If $(i)$ then $c(x)=c(y)$; if $(i i)$ then $c(y)=c\left(x_{i}\right)$. Regardless, $\Gamma \neq \emptyset$. Let $\delta \in \Gamma$. Set $A^{\prime}=\left\{x_{i-1}, x_{i+1}\right\}$ and $B^{\prime}=\left\{x_{i}\right\}$. Since Case 1 fails, $A^{\prime} \subseteq S-B$. Let $c^{\prime}\left(x_{i-1}\right)=\delta=c^{\prime}\left(x_{i+1}\right)$. Then $c_{b} \cup c^{\prime}$ is a proper coloring, since $\delta \in \Gamma$. The only possible components of $S-\left(A^{\prime} \cup B^{\prime}\right)$ with weight greater than 1 are the component that contains $x_{1}$ and the component that contains $x_{n-1}$. Regardless of whether $(i)$ or $(i i)$ hold, both $\left\{x, x_{i-1}\right\}$ and $\left\{x_{i+1}, y\right\}$ are well colored. So $c^{\prime}$ is good.

Finally suppose that for all $v \in B \cap P$, exactly one of $\{x, v\}$ and $\{v, y\}$ is not well colored. Then without loss of generality, $\left\{x, x_{i}\right\}$ is well colored (so $\left\{x_{i}, y\right\}$ is not). If $t \neq 0$ then set $A^{\prime}=\left\{x_{i+1}\right\}$ and $B^{\prime}=\left\{x_{i}\right\}$. Again $A^{\prime} \subseteq S-B$. Choose $c^{\prime}\left(x_{i+1}\right) \in X-c_{b}[B \cup\{y\}]$. Then $c_{b} \cup c^{\prime}$ is proper. There are only two new components of $S-\left(A^{\prime} \cup B^{\prime}\right)$ with weight 2 : the one that contains $x_{1} \ldots x_{i-1}$ and the one that contains $x_{i+2} \ldots x_{n-1}$. Both are well bounded since $\left\{x, x_{i}\right\}$ and $\left\{x_{i+1}, y\right\}$ are well colored. Thus $c^{\prime}$ is good. Otherwise $t=0$. Then $c\left(x_{i}\right)=c(y)$, since $\left\{x_{i}, y\right\}$ is not well colored. First suppose that $x_{i+2} \in B$. Then $\left\{x_{i+2}, y\right\}$ is well colored, since otherwise we would have preferred $x_{i+2}$ to $x_{i}$ either because $\left\{x, x_{i+2}\right\}$ is not well colored or because $\operatorname{dist}\left(x_{i+2}, y\right) \neq 0$. Let $A^{\prime}=\left\{x_{i+1}\right\}$ and $B^{\prime}=\left\{x_{i}, x_{i+2}\right\}$. Choose $c^{\prime}\left(x_{i+1}\right) \in X-c_{b}[B]$. Otherwise, $x_{i+2} \notin B$. Let $A^{\prime}=$ $\left\{x_{i+1}, x_{i+2}\right\}$ and $B^{\prime}=\left\{x_{i}\right\}$. Choose $c^{\prime}\left(x_{i+2}\right) \in X-c_{b}[B \cup\{y\}]$ and $c^{\prime}\left(x_{i+1}\right) \in$ $X-\left(c_{b}[B] \cup\left\{c^{\prime}\left(x_{i+2}\right)\right\}\right)$. Regardless of whether $x_{i+2} \in B^{\prime}$ or $x_{i+2} \in A^{\prime}$, the pair $\left\{x_{i+2}, y\right\}$ is well colored. So as above, $c^{\prime}$ is good. Moreover $c_{b} \cup c^{\prime}$ is proper.

Now we may assume that the internal vertices of $P$ are not in $B$.

Case 3. Cases 0,1 , and 2 fail, but there exist distinct vertices $u, v \in B$ such that $v \in Q_{u}$. Let $R$ be the union of the components of $S-\{v\}$ that do not meet $P$. Then $u \in R$. Let $S^{\prime}=S-R$ and $B^{\prime}=B \cap S^{\prime}$. So $v \in B^{\prime}$. Then $0<\left|B^{\prime}\right|<|B|$. By the induction hypothesis applied to $S^{\prime}$, there exists a set $A^{\prime} \subseteq S^{\prime}-B^{\prime}$, with $\left|A^{\prime}\right| \leq 2\left|B^{\prime}\right|$ and a good coloring $c^{\prime}: N \cup A^{\prime} \cup B^{\prime} \rightarrow X$ extending $c_{b} \mid N \cup B^{\prime}$. Clearly $c_{b} \cup c^{\prime}$ is a proper coloring, since no element of $B \cap R$ is adjacent to any element of $A^{\prime}$.

Case 4. Case $0,1,2$, and 3 fail, but there exist distinct $u, v \in B$ such that $Q_{u} \cap Q_{v} \neq \emptyset$. Choose $u, v$, and $w$ so that $w \in Q_{u} \cap Q_{v}$ and $w$ is as far from $P$ as possible. If possible, pick $u$ so that $u$ is not adjacent to $w$. Since Case 3 fails, $w \notin B$. Let $R$ be the union of the components of $S-\{w\}$ that do not meet $P$. Then 
$u, v \in R$. By the choice of $w$ and the fact that Case 3 fails, each component of $R$ contains at most one element of $B$.

First suppose that $u$ is not adjacent to $w$. Let $S_{u}$ be the component of $S-\{w\}$ that contains $u$. Let $S^{\prime}=S-S_{u}$ and $B^{\prime}=B-\{u\} \neq \emptyset$. By the induction hypothesis applied to $S^{\prime}$, there exists a set $A^{\prime} \subseteq S^{\prime}-B^{\prime}$, with $\left|A^{\prime}\right| \leq 2\left|B^{\prime}\right|$, and a good coloring $c^{\prime}: N \cup A^{\prime} \cup B^{\prime} \rightarrow X$ extending $c_{b} \mid N \cup B^{\prime}$. Since $u$ is not adjacent to any element of $A^{\prime}$, it follows that $c_{b} \cup c^{\prime}$ is a proper coloring.

Otherwise, by the choice of $u$, every vertex in $B \cap R$ is adjacent to $w$. This time we will find a good coloring $c_{a}$ that actually extends $c_{b}$. Let $S^{*}=S-R$ and $B^{*}=(B \cup\{w\})-(B \cap R)$. Let $\alpha$ be a color that is not used on any neighbor of $w$. Such a color exists because if $\Gamma$ is empty then one of $x$ and $y$ is not adjacent to $w$. Let $f: N \cup B^{*} \rightarrow X$ be the extension of $c_{b} \mid\left(N \cup B^{*}\right)$ defined by $f(w)=\alpha$. By the induction hypothesis applied to $S^{*}$ with $f$ playing the role of $c_{b}$, there exists a set $A^{*} \subseteq S^{*}-B^{*}$, with $\left|A^{*}\right| \leq 2\left|B^{*}\right|$, and a good coloring $c^{\prime}: N \cup B^{*} \cup A^{*} \rightarrow X$ extending $f$. Let $A=A^{*} \cup\{w\}$ and $c_{a}=c_{b} \cup c^{\prime}$. Then $|A| \leq 2\left|B^{*}\right|+1<2|B|$. Clearly $c_{a}$ is proper. Moreover, it is good, since $B \cap R \subseteq N(w)$, and so the uncolored components of $R-(A \cup B)$ have weight 1 .

Case 5. Cases $0,1,2,3$, and 4 fail. Then $B \cap P=\emptyset$ and $Q_{u} \cap Q_{v}=\emptyset$ for any distinct $u, v \in B$. Choose $z \in B \cap S$ such that, if $\operatorname{possible,~} \operatorname{dist}(z, P) \not 2$. Set $B^{\prime}=\{z\}$. Let $w$ be the endpoint of $Q_{z}$ in $P$. Since Case 2 fails, $w \notin B$. Since Cases 2 and 4 fail, $|N(v) \cap B| \leq 1$ for any $v \in P$. Moreover, $N(w) \cap(B-\{z\})=\emptyset$. Let $D=\{u \in\{x, y, z\}: \operatorname{dist}(u, w) \cong 2\}$. For each $u \in D$, let $u^{\prime}$ be the neighbor of $w$ on the $u-w$ path in $T$. Since Case 4 fails, $z^{\prime}$ (if it exists) has no neighbors in $B-\{z\}$.

First suppose that $|D| \leq 1$. Let $A^{\prime}=\left\{u^{\prime}: u \in D\right\} \cup\{w\}$. Then $\left|A^{\prime}\right| \leq 2\left|B^{\prime}\right|$. Since $b \geq 2$, we have $|X|=b+2 \geq 4$. Let $c^{\prime}(w)$ be any color in $X-c_{b}[\{x, y, z\}]$. If $u \in D$, let $c^{\prime}\left(u^{\prime}\right)$ be any color in $X-c_{b}\left[\left(N\left(u^{\prime}\right) \cap B\right)\right]$ distinct from $c^{\prime}(w)$ and $c_{b}(u)$. Then $c^{\prime}$ is good and $c_{b} \cup c^{\prime}$ is proper.

Otherwise $|D| \geq 2$. First suppose that $z \notin D$. Then $\operatorname{dist}(x, w) \cong 2 \cong \operatorname{dist}(w, y)$. So $\operatorname{dist}(x, y) \cong 1$. Since $\{x, y\}$ is well colored, $c(x) \neq c(y)$. Thus one of these colors, say $c(x)$, is distinct from $c_{b}(z)$. Let $A^{\prime}=\left\{w, y^{\prime}\right\}, c^{\prime}(w)=c(x)$, and $c^{\prime}\left(y^{\prime}\right) \in X-c_{b}\left[\left(N\left(y^{\prime}\right) \cap B\right) \cup\{x, y\}\right]$. Then $c^{\prime}$ is good and $c_{b} \cup c^{\prime}$ is proper.

Next suppose that $z \in D$. By the choice of $z$, for every $u \in B$, $\operatorname{dist}(u, P) \cong 2$. In particular, $N(v) \cap B=\emptyset$ for all $v \in P$. Without loss of generality, we may assume that $x \in D$. Choose a neighbor $w^{\prime}$ of $w$ on $P$ so that $\operatorname{dist}\left(w^{\prime}, y\right) \not 2$. Let $A^{\prime}=\left\{w^{\prime}, z^{\prime}\right\}$ and $c^{\prime}\left(w^{\prime}\right)=\delta=c^{\prime}\left(z^{\prime}\right)$, where $\delta \in X-c_{b}[\{x, y, z\}]$. Then $c_{b} \cup c^{\prime}$ is proper. We must check that $c^{\prime}$ is good. Here there is one additional complication. The component of $S-\left(A^{\prime} \cup B^{\prime}\right)$ that contains $w$ has three colored neighbors both $w^{\prime}$ and $z^{\prime}$ and either $x$ or $y$. However, $w^{\prime}$ and $z^{\prime}$ are both adjacent to the same vertex $w$ and are both colored with the same color $\delta$. Thus the weight of this component is only 2. It now follows, using the choice of $w^{\prime}$, that $c^{\prime}$ is good.

This completes the proof of Lemma 5. 


\section{LOWER BOUNDS}

In this section, we prove lower bounds on $\chi_{\mathrm{g}}(\mathcal{F} ; a, b)$ and $\operatorname{col}_{\mathrm{g}}(\mathcal{F} ; a, b)$. Let $T=T_{n}$ be the forest on the vertex set $V=\bigcup_{i=1}^{20}[n]^{i}$ whose edges are pairs of the form $e=\left(a_{1}, \ldots, a_{i}\right)\left(a_{1}, \ldots, a_{i+1}\right)$. Then every non-leaf has degree $n$ or $n+1$. All our proofs will show that Bob can win the appropriate game on $T$ if $n$ is sufficiently big. More precisely, we will show that for some $m$ Bob can win in $m$ turns playing on $T_{n}$ whenever $n \geq m(a+b)$. In this case, at the start of Bob's $m$ th turn, every non-leaf will have at least $b$ uncolored neighbors. In the proofs that follow we will explicitly calculate the number of turns $m$ that Bob uses to win, but implicitly assume that $n \geq m(a+b)$. For $i \in[n]$, we call the vertex $(i)$ a root of $T$. Notice that each component of $T$ has radius 20 with a root for its center. Bob will always play relatively close to the center. In particular, whenever our strategy calls for Bob to color a neighbor of a vertex $v$, it will be the case that $v$ is not a leaf.

When proving lower bounds on $\chi_{\mathrm{g}}(\mathcal{F} ; a, b)$, we will use the following concept. Suppose that at some point in the $(a, b)$-coloring game the players have created a partial coloring $c: C \rightarrow X$, where $X$ is the set of colors used for the game. For any vertex $y$, define the weight $w(y)$ of $y$ by $w(y)=|\{c(z): z \in N(y)\}|$. So Bob will win if $w(y)=|X|$ for some vertex $y$.

Lemma 7. For all positive integers $a$ and $b, b+2 \leq \chi_{\mathrm{g}}(\mathcal{F} ; a, b)$.

Proof. We argue that Bob can win the $(a, b)$-coloring game on $T$ with the set $X=[b+1]$ of colors in two turns. After Alice's first turn, Bob colors a root $x$ with $b+1$ and colors $b-1$ other vertices arbitrarily. After Alice's response, let $y$ be an uncolored neighbor of $x$. For each color $\alpha \in[b]$, Bob colors an uncolored neighbor of $y$ with $\alpha$. Then $w(y)=b+1$.

As remarked in the Introduction, Bodlaender proved that $4 \leq \chi_{\mathrm{g}}(\mathcal{F})=$ $\chi_{\mathrm{g}}(\mathcal{F} ; 1,1)$. The following lemma also yields this fact.

Lemma 8. $4 \leq \chi_{\mathrm{g}}(\mathcal{F} ; 2,1)$.

Proof. We will show that Bob can win the $(2,1)$-coloring game on $T$ using the set $X=$ [3] of colors in 13 turns. First we identify some winning positions for Bob in this game. These claims are valid for positions relatively close to the center.

Position 0. It is Bob's turn and $w(v) \geq 2$ for some uncolored vertex $v$. Then Bob wins in one turn by coloring an uncolored neighbor of $v$ with the third color.

Position 1. There is an uncolored path $P=v_{1} v_{2} v_{3}$ and a color $\alpha \in X$ such that each $v_{i}$ is adjacent to a vertex colored $\alpha \in X$. Note that if Alice colors one or two of the vertices in $\left\{v_{1}, v_{2}, v_{3}\right\}$ then she must leave Position 0. Otherwise Bob wins by coloring a neighbor of $v_{2}$ with a color other than $\alpha$. After Alice's next play, this leaves Position 0. So Bob wins in two turns. 
Position 2. It is Bob's move and there is an uncolored path $P=v_{1} v_{2} v_{3}$ and a color $\alpha \in X$ such that at least two vertices of $P$ have a neighbor colored $\alpha$. Then Bob produces Position 1 by coloring a neighbor of a vertex in $P$ so that every vertex in $P$ has a neighbor colored $\alpha$. So Bob wins in three turns.

Position 3. There is an uncolored star $\left\{s, v_{1}, v_{2}, v_{3}\right\}$ with leaves $v_{1}, v_{2}, v_{3}$ such that for each $i \in[3], v_{i}$ is adjacent to a vertex colored $i$. Note that if Alice colors $s$ then she must leave Position 0. Otherwise, if Alice colors a vertex in $\left\{v_{1}, v_{2}, v_{3}\right\}$, then she must leave Position 0 or 2. So Alice does not color any of the vertices in $\left\{s, v_{1}, v_{2}, v_{3}\right\}$. On his next turn Bob wins by coloring a neighbor of $v_{1}$ distinct from $s$ with 2. Regardless of how Alice plays, she must leave Position 0 or 2. So Bob wins in four turns.

Position 4. It is Bob's move and there is an uncolored path $v_{1} s v_{2}$ such that for each $i \in[2], v_{i}$ is adjacent to a vertex colored $\alpha_{i}$ and $\alpha_{1} \neq \alpha_{2}$. Let $v_{3} \in N(s) \cap$ $U-\left\{v_{1}, v_{2}\right\}$ and $u \in\left(N\left(v_{3}\right) \cap U\right)-\{s\}$. Then Bob creates Position 3 by coloring $u$ with $\alpha_{3} \in X-\left\{\alpha_{1}, \alpha_{2}\right\}$. So Bob wins in five turns.

We now describe Bob's strategy. On his first turn, Bob colors a root $y_{1}$. After Alice's response, Bob chooses a path $P=y_{1} \ldots y_{11}$ in $T$ such that the vertices $y_{2}, \ldots, y_{11}$ are uncolored. Then he colors $y_{11}$. After Alice plays, there exists a $y_{i}-y_{j}$ subpath $P^{\prime}=y_{i} \ldots y_{j}$ of $P$ such that the endpoints of $P^{\prime}$ are colored, the internal vertices of $P^{\prime}$ are uncolored, and $\left|P^{\prime}\right| \geq 5$. We shall show by induction on $t=\left|P^{\prime}\right|$ that Bob can win from this position in $t$ turns.

First consider the base step $t=5$. If $y_{i}$ and $y_{j}$ have different colors then we have Position 4. Otherwise $y_{i}$ and $y_{j}$ have the same color $\alpha$ and we have Position 2. In either case, Bob wins in five turns. Next consider the induction step. Let $u$ be an uncolored neighbor of $y_{i+1}$, which is different than $y_{i+2}$. Bob colors an uncolored vertex $v \in N(u)-\left\{y_{i+1}\right\}$ with the same color as $y_{i}$. To avoid Position 2, Alice must color $y_{i+1}$ or $u$. But then to avoid Position 0, she must color both $y_{i+1}$ and $u$. Then by the induction hypothesis, Bob can win in $t-1$ additional turns. We conclude that Bob can win in $2+t \leq 13$ turns.

Lemma 9. If $a<2 b$, then $b+3 \leq \chi_{\mathrm{g}}(\mathcal{F} ; a, b)$.

Proof. We will show that Bob can win the $(a, b)$-coloring game on $T$ using the set $X=[b+2]$ of colors in $2 b+3$ turns. Call an uncolored vertex dangerous if it has weight at least 2. Bob's plan is to force Alice to leave a dangerous vertex $x$ at the end of one of her turns. He can then win by coloring $b$ neighbors of $x$ so that $w(x)=b+2$.

On his first turn, Bob colors some root $x$ with $\alpha$. The first round of play ends after Alice completes her second turn. On his next $2 b$ turns, Bob colors vertices $v_{1}, \ldots, v_{2 b^{2}}$ with color $\alpha$, where $\operatorname{dist}\left(x, v_{i}\right)=3$, $\operatorname{dist}\left(v_{i}, v_{j}\right)=6$ for all distinct $i, j \in\left[2 b^{2}\right]$, and before Bob colors $v_{i}$, the only colored vertex on the $x-v_{i}$ path in $T$ is $x$. Let $\mathcal{P}$ be the set of $x-v_{i}$ paths such that $i \in\left[2 b^{2}\right]$. After Alice's response, the second round of play ends. 
During the second round, Alice colored at most

$$
2 b a \leq 2 b(2 b-1)=4 b^{2}-2 b
$$

of the $4 b^{2}$ internal vertices of paths in $\mathcal{P}$. Thus at the start of the third round, there exists a $b$-subset $\mathcal{P}^{\prime} \subseteq \mathcal{P}$ such that each path $P \in \mathcal{P}^{\prime}$ has an uncolored (internal) vertex $y$. If $y$ is the only uncolored vertex in $P$ then $y$ is dangerous and Bob wins. Otherwise both internal vertices of each path in $\mathcal{P}^{\prime}$ are uncolored. On his next turn, for each $P \in \mathcal{P}^{\prime}$, Bob colors an external neighbor of an internal vertex $y \in P$ with a color $\beta \neq \alpha$. This makes $y$ dangerous. Alice is now stuck. On her next turn, she must color each of $b$ dangerous vertices in $\cup \mathcal{P}$. But in doing so she creates $b$ additional dangerous vertices in $\cup \mathcal{P}$ and she can color less than $2 b$ vertices in all.

Lemma 10. If $a<b$ then $\chi_{\mathrm{g}}(\mathcal{F} ; a, b)>t$ for every integer $t$.

Proof. We will show that Bob has a strategy for the $(a, b)$-coloring game played on $T$ with the set $X=[t]$ of colors that wins in a fixed number of moves. Actually we will prove a stronger statement. For natural numbers $s$ and $t$, let $P(s, t)$ mean that there exists an independent $s$-set $S \subseteq U$ of non-leaves such that $w(y) \geq t$ for all $y \in S$. We shall show that for all natural numbers $s$ and $t$, there exists a natural number $m$ for which Bob has a strategy that produces a position for which $P(s, t)$ holds after $m$ turns. We argue by induction on $t$. The base step $t=0$ is trivial, so consider the induction step $t=t^{\prime}+1$. Let $s^{\prime}=s b$. Then there exists $m^{\prime}$ such that Bob can play so that after $m^{\prime}$ turns, $P\left(s^{\prime}, t^{\prime}\right)$ holds. Let $m=m^{\prime}+s$. Suppose that Bob has played so as to obtain an $s^{\prime}$-set $S^{\prime}$ that witnesses $P\left(s^{\prime}, t^{\prime}\right)$. For his next $s$ moves, Bob colors exactly one neighbor $y^{\prime}$ of each vertex $y \in S^{\prime}$ with a color $c(y)$ such that if $w(y)<t$ then $c(y)$ has not yet been used on a neighbor of $y$. So now $w(y) \geq t^{\prime}+1=t$ for every vertex $y \in S^{\prime}$. During this time, Alice colors at most as vertices of $S^{\prime}$. Thus after $m$ turns $\left|S^{\prime}-C\right| \geq(b-a) s \geq s$ where $C$ is the set of colored vertices. So $S^{\prime}-C$ witnesses $P(s, t)$.

Lemma 11. If $a<3 b$, then $b+3 \leq \operatorname{col}_{\mathrm{g}}(\mathcal{F} ; a, b)$.

Proof. We will show that Bob can obtain a score of $b+2$ in the $(a, b)$ marking game on $T$ in $9 b^{2}+3 b+2$ turns. Call an unmarked vertex dangerous, if it has two marked neighbors. Bob's plan is to force Alice to leave a dangerous vertex $x$ at the end of one of her turns. He can then obtain a score of $b+2$ by marking $b$ additional neighbors of $x$.

On his first turn, Bob marks a root $x$. The first round of play ends after Alice completes her second turn. At the start of the second round, Bob chooses a $9 b^{3}$ set $\mathcal{P}$ of internally disjoint paths such that for each $P \in \mathcal{P}$ :

- $|P|=5$.

- $x$ is an endpoint of $P$.

- $P-\{x\} \subseteq U$, where $U$ is the set of unmarked vertices. 
On his next $9 b^{2}$ turns, Bob marks all the (still) unmarked endpoints of the paths in $\mathcal{P}$, but no other points on these paths. After Alice's response, the second round of play ends.

During the second round, Alice marked at most

$$
9 b^{2} a \leq 9 b^{2}(3 b-1)=27 b^{3}-9 b^{2}
$$

of the $27 b^{3}$ internal vertices on the paths in $\mathcal{P}$. Thus at the start of the third round of play, there exists a $3 b^{2}$-subset $\mathcal{P}^{\prime} \subseteq \mathcal{P}$ such that for all $P \in \mathcal{P}^{\prime}$, at least one of the (internal) vertices of $P$ is unmarked. If $P$ does not have two consecutive unmarked vertices then $P$ has a dangerous vertex and we are done. So suppose each $P \in \mathcal{P}^{\prime}$ has at least two consecutive unmarked vertices. On his next $3 b$ turns, Bob plays as follows. For each $P \in \mathcal{P}^{\prime}$ with three unmarked vertices, Bob marks an external neighbor of the central vertex of $P$. For each $P \in \mathcal{P}^{\prime}$ with only two (consecutive) unmarked vertices, Bob marks an external neighbor of an unmarked, external neighbor of the central vertex of $P$. In either case we are left with a path $P_{0} \subseteq U$ on three vertices such that each vertex of $P_{0}$ has a marked neighbor. Let $\mathcal{P}_{0}=\left\{P_{0}: P \in \mathcal{P}^{\prime}\right\}$. The third round ends after Alice's response.

During the third round, Alice marks

$$
3 b a \leq 3 b(3 b-1)=9 b^{2}-3 b
$$

vertices. If she marks a vertex of a path $P^{\prime} \in \mathcal{P}_{0}$ then she must mark all three vertices of $P^{\prime}$, since otherwise she will leave a dangerous vertex. Thus at the start of the fourth round of play, there exists a $b$-subset $\mathcal{P}_{0}^{\prime} \subseteq \mathcal{P}_{0}$ such that for every $P^{\prime} \in \mathcal{P}_{0}^{\prime}$ all three vertices of $P^{\prime}$ are unmarked. On his next turn, Bob plays by marking an external neighbor of the central vertex of $P^{\prime}$ for each $P^{\prime} \in \mathcal{P}_{0}^{\prime}$. So for each path $P^{\prime} \in \mathcal{P}_{0}^{\prime}$, the central vertex is dangerous. Alice is now stuck. She must mark each of the $b$ dangerous central vertices that Bob has left. However this creates $2 b$ additional dangerous vertices and she can mark at most $3 b-1$ vertices on any one turn.

This completes the proof of Theorem 1 .

\section{REFERENCES}

[1] H. L. Bodlaender, On the complexity of some coloring games, Graph Theoretical Concepts in Computer Science, R. H. Möhring, (Editor), Vol. 484 of Lecture Notes in Computer Science, 30-40, Springer, 1991.

[2] L. Cai and X. Zhu, Game chromatic index of $k$-degenerate graphs, J Graph Theory 36 (2001), 144-155.

[3] T. Dinski and X. Zhu, A bound for the game chromatic number of graphs, Discrete Math 196 (1999), 109-115. 
[4] U. Faigle, U. Kern, H. A. Kierstead, and W. T. Trotter, On the game chromatic number of some classes of graphs, Ars Combinatorica 35 (1993), $143-150$.

[5] D. Guan and X. Zhu, Game chromatic number of outerplanar graphs, J Graph Theory 30 (1999), 67-70.

[6] H. A. Kierstead, A simple competitive graph coloring algorithm, J Comb Theory (B) 78 (2000), 57-68.

[7] H. A. Kierstead and W. T. Trotter, Planar graph coloring with an uncooperative partner, J Graph Theory 18 (1994), 569-584.

[8] H. A. Kierstead and W. T. Trotter, Competitive colorings of oriented graphs, Electron J Combina 8(12) (2001), 15 pp. (electronic).

[9] H. A. Kierstead and Zs. Tuza, Marking games and the oriented game chromatic number of partial $k$-trees, Graphs and Combinatorics 19 (2003), $15,121-129$.

[10] J. Nešetřil and E. Sopena, On the oriented game chromatic number, Electro J Combin 8(14) (2001), 13 pp. (electronic).

[11] X. Zhu, Game coloring number of planar graphs, J Combin Theory (B) (1998), 245-258.

[12] X. Zhu, Game coloring number of pseudo partial $k$-trees, Discrete Math 215 (2000), 245-262. 